\title{
A New Agar Plate Assisted Slide Culture Technique to Study Mycoparasitism of Trichoderma sp. on Rhizoctonia solani and Fusarium oxysporium
}

\author{
K. A. Bhat ${ }^{*}$ \\ Division of plant Protection, Faculty of Agriculture Wadura, Sher-i-Kashmir University of \\ Agricultural Sciences and Technology of Kashmir, India \\ *Corresponding author
}

\section{A B S T R A C T}

Keywords

Mycoparasitism, in-vitro, Technique, Trichoderma, Plant pathogens

Article Info

Accepted:

26 June 2017

Available Online:

10 August 2017
Mycoparasitism by Trichoderma $s p$. is one of the important modes of action exhibited by biocontrol agents against host fungi including plant pathogenic ones. Its study in-vitro constitutes considerable importance while studying bio-efficacy of an antagonist against a fungal pathogen. At present there is no standard, rapid, easy and reliable method to study mycelial interaction between a possible mycoparasitic fungi and host. An easy, cheap, and rapid agar plate assisted slide culture technique is developed which facilitates the study of in-vitro mycoparasitism with ease. With the help of this technique mycoparasitism by a strain of Trichoderma sp. was studied against pathogenic Rhizoctonia solani and Fusarium oxysporium. A drastic mycoparasitism in the form of coiling and tightly sticking with occasional formation of appressoria like structures was observed while studying mycoparasitism with the help of this method.

\section{Introduction}

Mycoparasitism consists of a direct attacks by a fungus to another and leads to the destruction of some of the structures of the host (e.g. mycelium, spores and sclerotia) with the consequent harnessing of their components as a nutrient source. The process of mycoparasitism exerted by amycoparasite e.g Trichoderma sp. occurs in several successive stages. Starts with the chemo trophic growth of Trichodermato the host stimulated by molecules from the same (Chat, et al., 1981). When Trichoderma comes into contact with the host are cognition of this must occur that triggers the following steps. After the recognition occurs the coiling or sticking of Trichoderma around the host hyphae for ming gaff structures or shaped like appressoria (Elad, et al., 1983). After this, Trichoderma secrethydrolytic enzymes primarily chitinases, glucanases and proteases, which degrade the cell wall of the host (Haran et al., 1996) with the consequent assimilation of the components of the cell wall and cellular contents thereof (Elad et al., 1984). Mycoparasitism is being considered as a mechanism of biocontrol by various fungal antagonists like Trichoderma spp and $G$. virens and many other antagonistic fungi. As far mycoparasitism as one of the important mechanisms of action of a fungal biocontrol 
agent is concerned, the study of mycoparasitic potential against a pathogen(s) in-vitro carries a lot of significance. To study mycoparasitism in-vitro workers use several methods as there being no standard method which is easy, rapid, reliable and accurate.

The present paper pertains to a simple, easy and new method to study mycoparasitic potential of a biocontrol agent Trichoderma sp. against plant pathogens viz., $R$. solini and Fusarium oxysporium potent pathogens causing root rot and wilt in common beans invitro.

\section{Materials and Methods}

The method involves easy steps under strict aseptic conditions described as follows.

A $90 \mathrm{~mm}$ petri dish was poured with Potato Dextrose Agar (PDA) only upto the thickness of $3-4 \mathrm{~mm}$ roughly around the thickness of a glass slide and allowed to solidify.

A sterilized glass slide was placed on the solidified agar medium and gently pressed so as to leave the impression of glass slide on the PDA medium. Thereafter the slide was removed (Plate-1a).

The agar medium was cut with a sterilized blade as per the impression left by glass slide previously pressed on it so that an agar strip exactly equivalent to dimensions of glass slide was removed from the petri plate (Plate1b).

A sterile glass slide is fitted into the hollow strip in such a way that all its sides will touch the PDA and the upper surface of glass slide remains in uniformity with the surface of PDA (Plate-1c).

Host fungi was inoculated at various points on PDA on the edges of glass slides (Plate-1d).
The plate was incubated for 36 hours allowing the host fungi to grow partly on the medium as well as on glass slide.

After appearance of growth of Host mycelium on slide edges bioagent Trichoderma sp was inoculated on edges of glass slide in the vicinity where previously host fungi was inoculated and the petri plate is incubated again for 24 hours.

When the two fungai visibly interacted a sharp razor was carefully run all around the glass slide so that all the mycelium mats were cut between the slide and agar surface without disturbing the interacting fungal growth/mat on the glass slode. The slide was carefully removed without disturbing fungal growth on the slide. The under surface of slide was cleaned with the help of bloating paper. The slide was stained gently with cotton blue stain and viewed directly under microscope for hyphal interaction between the bioagent and host fungi.

\section{Results and Discussion}

After incubation of 24 to 36 hours of bioagent inoculation, the slides were gently removed as described in materials and methods. The slides were stained and directly observed under microscope both with and without coverslip as well as at low and high power. The microscopic potential of Trichoderma sp. against R. solani and Fusarium oxysporium was clearly revealed. The parasitic fungi Trichoderma sp grew in close contact with $R$. solini with occasional appressoria like structures seen penetrating $R$. solini. (Plate$2)$.Frequent coiling by hyphae of Trichoderma sp. was also seen around $R$. solini. (Plate-3). Similarly hyphal interaction between Trichoderma sp. and Fusarium oxysporium as observed by above mentioned technique revealed drastic mycoparasitism by Trichoderma sp. including tightly growing 
along the host hyphae as well as coiling around host hyphae.

Workers have used different methods for studying of in-vitro mycoparasitism. In one method cellophane membrane is used over a agar plate on which dual culture is conducted afterwards placed over the glass slide for observation (El-Naggar et al., 2008). This method is tedious, expensive and visibility during microscopy is hindered to a larger extent, besides the cellophane membrane over the glass slide may hinder observation under high power. Most other workers used Mycelial samples, cut from the interaction region from dual-culture plates.
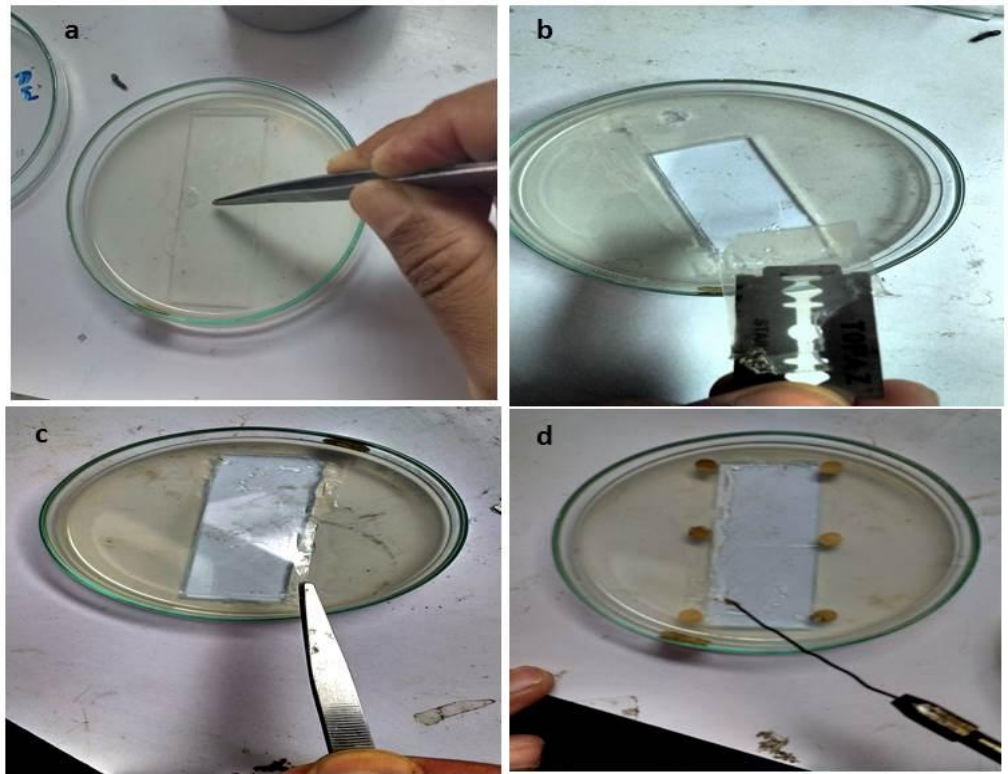

Plate-1: Agar plate assisted Slide Culture Technique a) a slide pressed on agar surface. b) an agar strip equivalent to glass slide cut from the agar plate. c) Glass slide fitted into hallow strip. d) Inoculation of pathogen on edges of glass slide
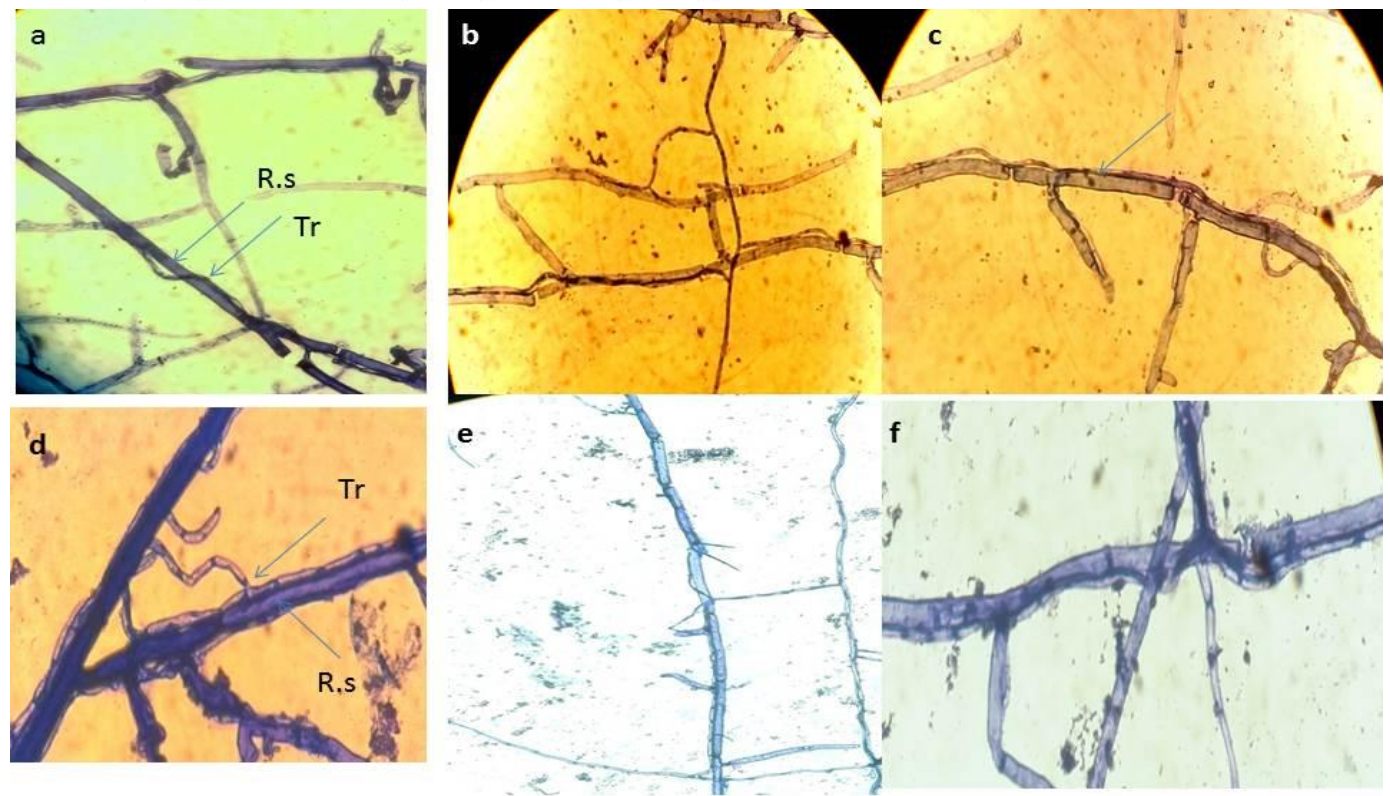

Plate-2: a-b) Sticking and growing of Trichoderma sp. along R. solini.x40 c) Tightly sticked hypha of Trichoderma with appressoria like projections on $R$. solini. d) Parssitism of $R$. solini by Trichoderma $s p$. from both sides $\times 100 . \mathrm{e}-\mathrm{f})$ tightly attached bio agent Trichoderma $s p$. with host $\mathrm{x} 40$ 

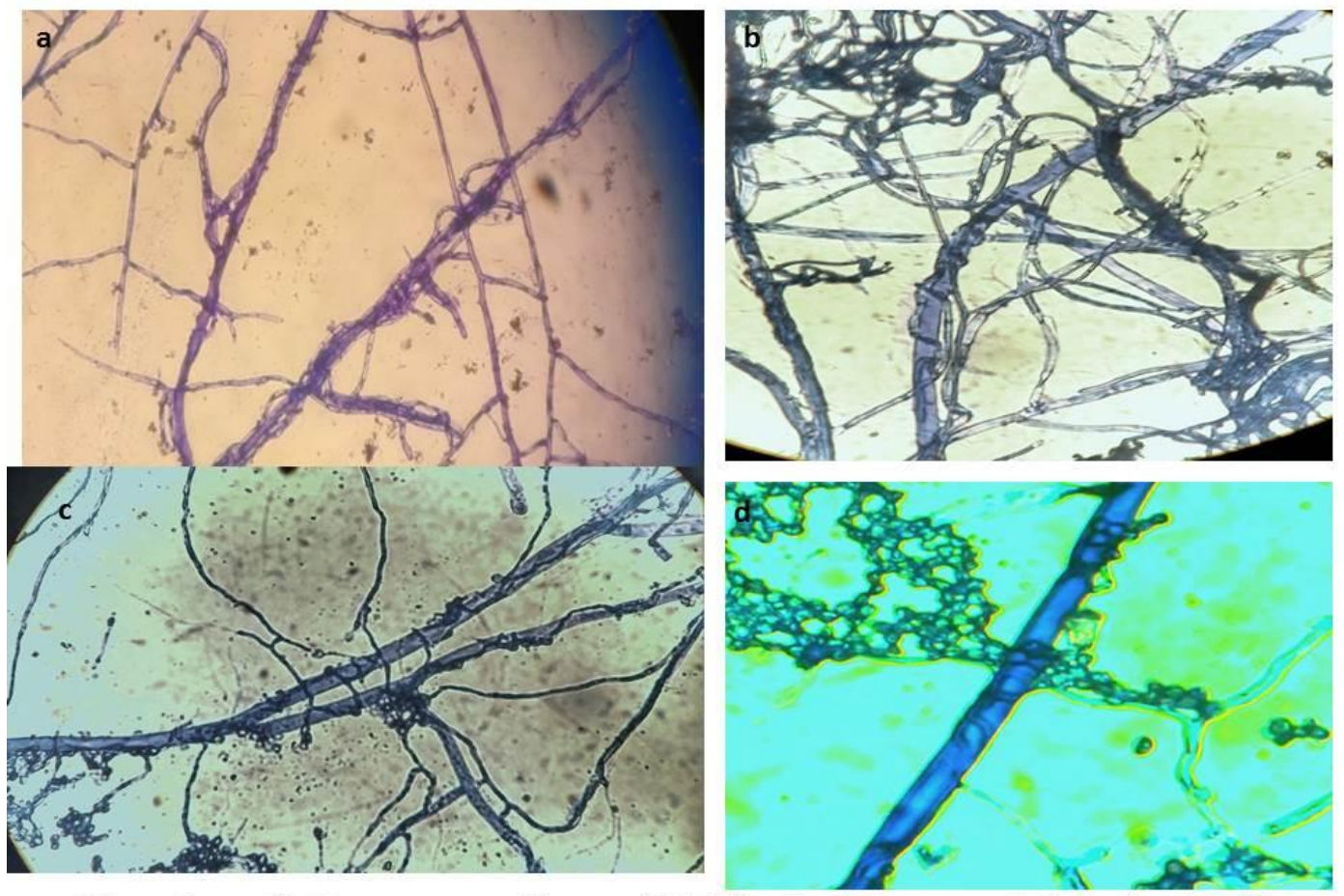

Plate-3: a-d) Frequent coiling of Trichoderma sp. and $R$. solani $\mathrm{x} 40$

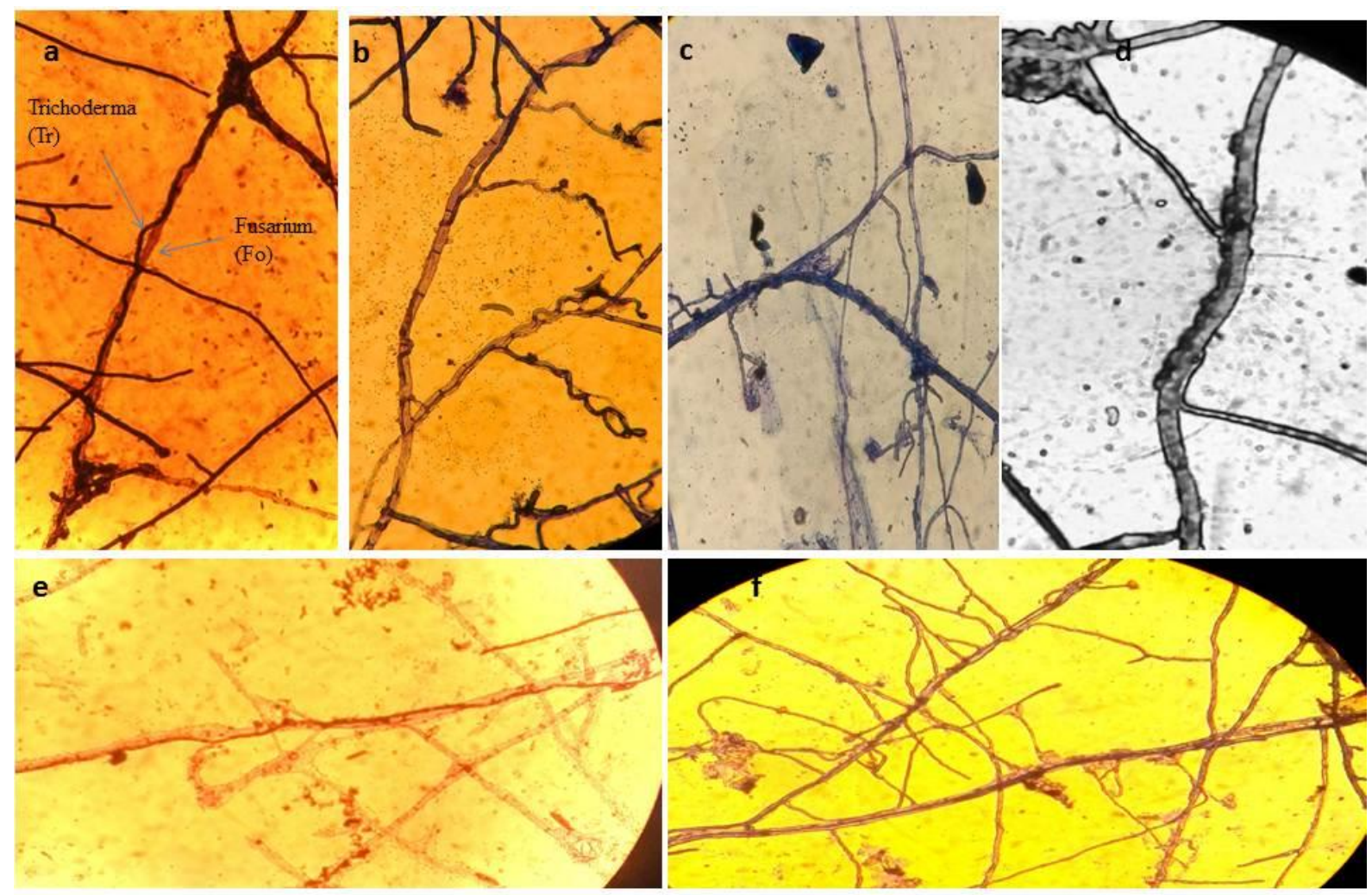

Plate-4: a-f) Mycoparasitism between Trichoderma sp. and Fusarium oxysporium $\mathrm{x} 40$ 
These mycelial samples are fixed on slide and observed under an inverted binocular light microscope for the presence of coiling and other types of parasitism (Ronghua, et al., 2009; Gajera et al., 2012). This method is also very tedious. Observation of mycoparasitism by this method is often not very clear as while picking up the mycelium they get distorted and mingled and mycoparasitism is not visible in its original form. In one more technique used by various researchers, in which a microscopic slide is covered with thin layer of medium followed by inoculation of pathogen and bioagent discs on opposite sides, followed by incubation of slide encased in a petri dish (Mohamed, 2015). By this technique although the interacting mycelium may not disturb much but due to presence of agar medium along with mycelium the visibility during microscopy is not clear, besides observation of mycoparasitism under high power also becomes difficult. In all above methods and many others suffer from many shortcomings manin ones being that hyphal interaction between a antagonist and host fungi is not clearly observed as the same is disturbed while removing the interacting mycelium from the cultures, besides there is intermingling of hyphae. Second being that, visibility is not clear. In present innovative technique, the interaction of two fungi is on the clean glass slide, hence can be observed under any power of compound microscope. The staining is easy and there is no any form of mixing or intermingling of mycelium during removal of mats from dual cultures as the two interacting mycelia are not disturbed but viewed as such. This technique is a handy method to study possible mycoparasitism between fungal antagonists and host fungi.

\section{References}

Chet, I., Harman, G.E., Baker, R. (1981) Trichoderma hamatum: Its hyphal interactions with Rhizoctonia solaniand Pythium spp. Microb Ecol 7: 29-38.

EL-Naggar, Magdy, Kovics, György Janos., Sandor, Erzsebet. And, Irinyi Laszlo. (2008). Mycoparasitism and Antagonistic Efficacy of Trichoderma reesei Against Botrytis spp. Contribuţii Botanice, Grădina Botanică, Alexandru Borza, Cluj-Napoca, 18:141-147

Elad, Y., Chet, L., Boyle, P., Henis, Y. (1983) Parasitism of Trichoderma spp. on Rhizoctonia solani and SclerotiumrolfsiiScanning electron microscopy and fluorescence microscopy. Phytopathology 73: 85-88.

Elad, Y., Barak, R., Chet, L. (1984) Parasitism of sclerotia of Sclerotiumrolfsiby Trichoderma harzianum. Soil BiolBiochem 16: 381-386.

Gajera, H.P., Bambharolia, R.P., Patel, S.V., Khatrani, T.J., and Goalkiya, B.A. (2012). Antagonism of Trichoderma spp. against Macrophominaphaseolina: Evaluation of Coiling and Cell Wall Degrading Enzymatic Activities. Plant PatholMicrob.3:7:1000149

Haran, S., Schickler, H., Chet, L. (1996) Molecular mechanisms of Iytic enzymes involved in the biocontrol activity of Trichoderma harzianum. Microbiology 142: 2321-2331.

Mohamed, A, Mahmoud, (2015). Efficiency of some Bioagents and Nemastop compound in controlling damping off and root rot diseases on peanut plants. Int. J. Adv. Res. Biol. Sci. 2(11): 77-86

Ronghua,Cao.,Xiaoguang, Liu., Kexiang, Gao., Kurt Mendgen., Zhensheng, Kang., Jianfeng, Gao., Yang, Dai., and Xue, Wang. (2009).Mycoparasitism of Endophytic Fungi Isolated from Reedon Soil borne Phytopathogenic Fungi and Production of Cell Wall-Degrading Enzymes in Vitro. Curr Microbiol: DOI 10.1007/s00284-0099477-9

\section{How to cite this article:}

Bhat, K.A. 2017. A New Agar Plate Assisted Slide Culture Technique to Study Mycoparasitism of Trichoderma sp. o on Rhizoctonia solani and Fusarium oxysporium. Int.J.Curr.Microbiol.App.Sci. 6(8): 3176-3180. doi: https://doi.org/10.20546/ijcmas.2017.608.377 